\title{
A Scrutiny Of Self Restraint Of Collision Using Gps \& Gsm Tracking System
}

\author{
Fathima Zaheera, P. Maniganda, B. Dharmendar, K. Rajesh, K. Srikanth
}

\begin{abstract}
As a breeding populace, it produces to a large quantity of automobiles on the road and consequently ends in growth within the problems associated with site site visitors manage. On this paper, we recommend a monitoring device based totally mostly on numerous standards including smart delivery systems (ITS), satellite tv for pc television for laptop modem, RFID ( radio frequency identity), Raspberry pi, GPS, GPRS, GSM, and lots of others. With this start we will reveal vehicle monitoring structures the use of GPS systems. The primary motivation of this art work is to introduce the idea of the above stated device is an revolutionary enhancement to allow people to revel in flexibility in motion and advanced a complex sense of self assurance and self-monitoring.
\end{abstract}

Key terms - ITS, RFID, GPS GPRS, GSM, Raspberry, pi, satellite modem

\section{INTRODUCTION}

GPS deposit the current vicinity of the car, extinguish GPRS monitoring statistics to a server and GSM is used to send a warning message to the car proprietor's cellular phone. This paper turned into prepared as described further my aspect- provide precise literature survey on the mixing of clever telephones and IOT to the improvement of public delivery clever machine.Section- II is an Arduino based implementation.Phase-III to preserve with a brief description the use of satellites. Part IV and section V affords RFID and raspberry pi implementation of the proposed shape

\section{SURVEY OF LITERATURE}

A. The combination of clever telephones and IOT for the development of an sensible public delivery gadget

Shrewd transportation structures (ITS) provides a method to this problem with the appearance of cell era and ubiquitous network innate, real time vehicle tracking for an green shipping control has become viable [1] by using a combination of GPS and Android that offer pride for the cruise passengers through public delivery.From proposed device, a smooth android and IOT-based approach which

Revised Manuscript Received on September 14, 2019.

Fathima Zaheera, Assist. Prof, Department of ECE, Siddhartha Institute of Technology \& Sciences, Narapally, Ghatkesar, Hyderabad, Telangana, India.

P. Maniganda, Associate Prof, Department of ECE ,Affliated to JNTU colleges, (Email: manigandapuraji@gmail.com)

B. Dharmendar, Assist. Prof, Department of ECE, Siddhartha Institute of Technology \& Sciences, Narapally, Ghatkesar, Hyderabad, Telangana, India.

K. Rajesh, Assist. Prof, Department of ECE, Siddhartha Institute of Technology \& Sciences, Narapally, Ghatkesar, Hyderabad, Telangana, India.

K. Srikanth, Assist. Prof, Department of ECE, Siddhartha Institute of Technology \& Sciences, Narapally, Ghatkesar, Hyderabad, Telangana, India could provide tracking information for a dynamic bus save you and a commuter bus in an efficient way.

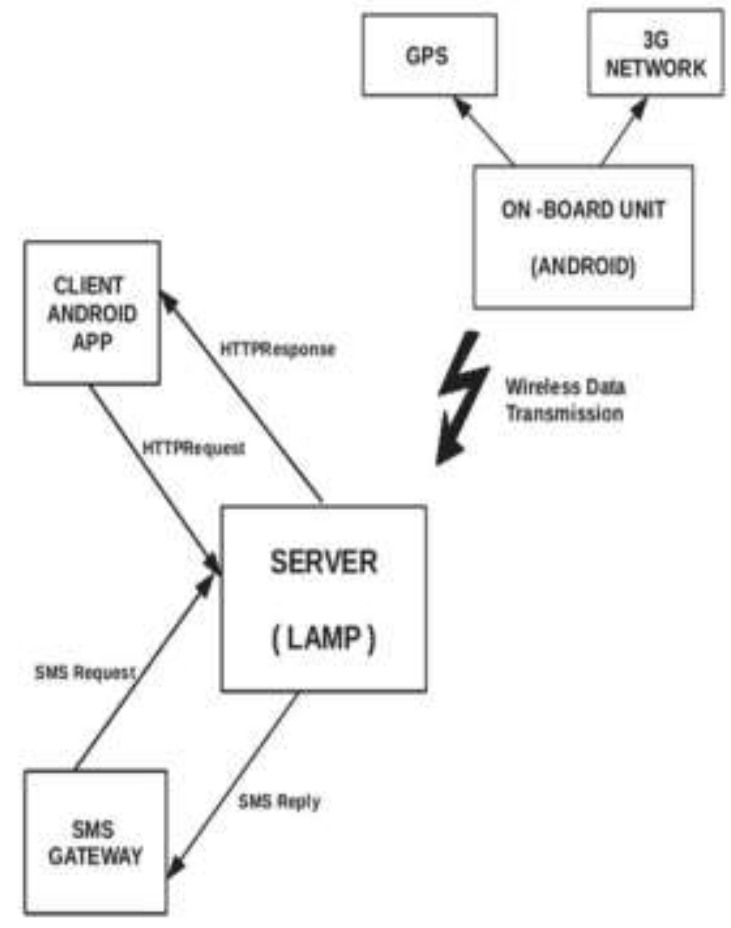

Fig. 1. Square graph proposes an exorbitant level perspective on the machine

From fig 1, the engineering is part into parts (ie) on-board unit and a server on-board unit is particularly made out of GPS and $3 \mathrm{G}$ people group that is situated inside the vehicle to give certainties in manner.Whereas simpler and snappier, http server gets demands from clients through the Android App and answer to supporter mentioned has been gotten from the UNIT oN-BOARD through remote measurements transmission. The some other way to dispatch demands and answers are performed by methods for SMS entryway.

Further, mass travel has moved toward each in significant towns, making it simpler to make a trip to any piece of the city, making transport sly want to pick. It bears non-open versatility and opportunity for individuals from each walk around ways of life [1].

A. Continuous maps of Google and Arduino-based absolutely vehicle following machine

A vehicle following $\mathrm{s} / \mathrm{m}$ to give records of the area to each other on each event moments. In this paper, a Google guide and Arduino vehicle dependent on betting a prime job

Published By:
Blue Eyes Intelligence Engineering

\& Sciences Publication 
for frameworks which are actualized utilizing GPS and GSM observing. Specialists offer spot records, simultaneously as GSM transmit place realities in expressions of range and longitude with the guide of SMS is in like manner a vehicle principally dependent on the Arduino observing the S/M comprises of two sections: equipment and $\mathrm{S} / \mathrm{W}$

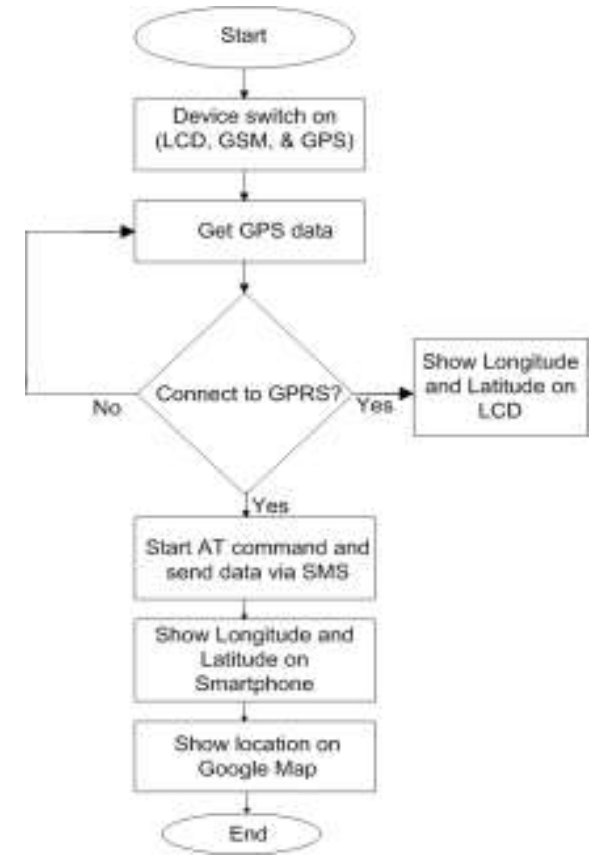

Fig2.represents the flowchart of the proposed tracking S/M.

Arduino based automobile monitoring device has two elements - hardware and software specifications. Hardware specification consists of an Arduino UNO board with a microcontroller, GPS and GPRS guard, LCD. In the specification of the software program, the software IDE Aurdino, Google maps are us. At first, 5 volt DC strength is provided to the GSM and GPRS shields, Arduino UNO board with a microcontroller to set off the tool. Arduino is used to manipulate the system with a GPS receiver and GSM module.GPSreceiver labored at 9600 baud charge this is used to receive statistics from the satellite. Data is despatched to the Arduino, which reads the coordinates by way of extracting \$ GPGGA String from a GPS receiver, and forwarded to GSM in phrases of coordinates (latitude and longitude). GSM module sends the coordinates to the person / proprietor with SMS so that he can tune vehicles round the sector, and watch the placement immediately on Google maps. And optional $16 \times 2 \mathrm{LCD}$ is also used to display coordinates. [2]

\section{UTILITY SYSTEM}

Low price Arduino based car monitoring machine can be extensively used in tracing the taxi / taxies, stolen motors, school / university buses and so forth. In addition, caregivers can without difficulty tune college youngsters away to keep away from accidents. If the device is equipped with a bus or educate, it will shop time and keep away from waiting for a long time passengers. One can also locate the closest taxi using this generation. This machine can be employed fleet management structures and shrewd transportation systems
(ITS), in addition to monitoring of human beings and animals inside the wooded area. It can be utilized by the shipping of meals and vehicle condo corporations as nicely.

\section{ADVANTAGES OF THE MACHINE \& RESULTS}

(I) For Arduino and use GPS and GPRS shield, lowering system complexity.

C. An innovation implementation of indoor positioning system the use of GPS

Global positioning S / M is used by GPS tracking gadgets to decide the precise place of the consumer. Analysts information may be transmitted to the database or tool related computers through radio, satellite modem embedded in the unit. Satellite navigation is basically a manner of several strategies of determining or planned positioning of items and of path with geometry. Worldwide navigation satellite tv for $\mathrm{pc}$ gadget offers users with a solution of three-dimensional position with a passive start the usage of radio alerts transmitted by orbiting satellites. This willpower function especially depends at the number of satellites to be had at a time. Availability depends at the constellation or satellite tv for pc geometry. A exact satellite geometry is obtained while the satellite absolutely dispersed within the sky [3].

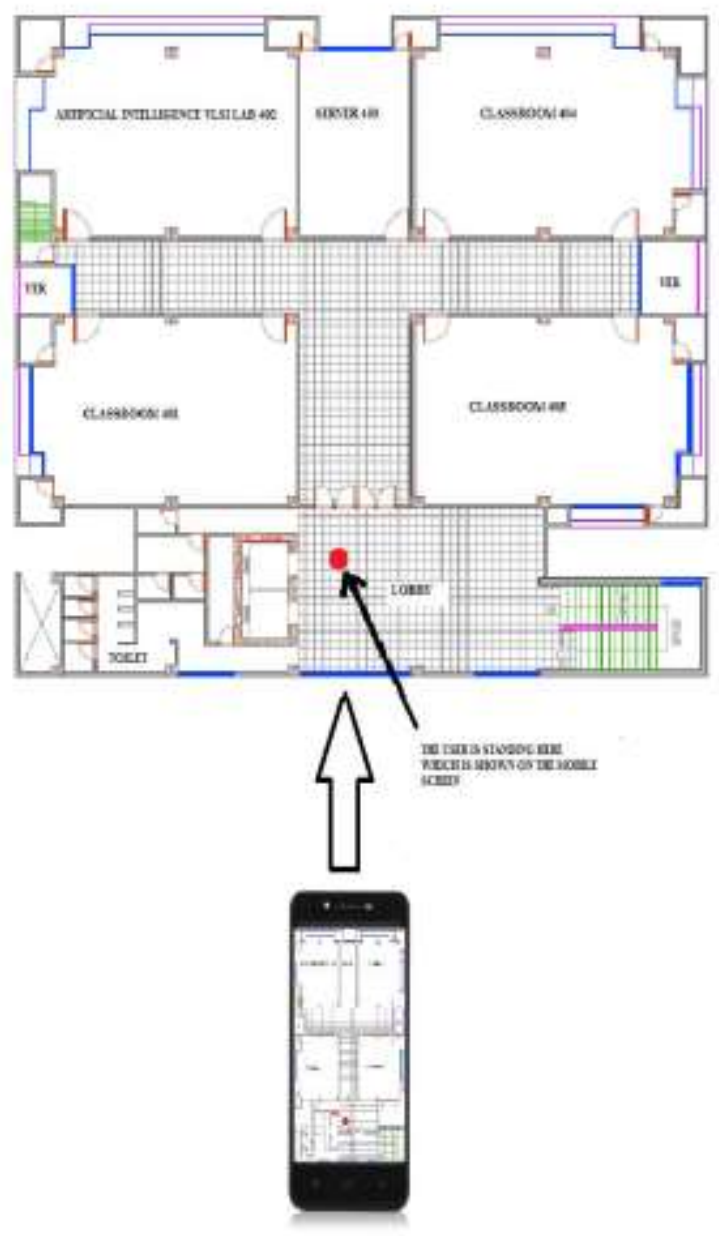

Fig.Three.The hypothetical output on the telephone show screen.

Published By: Blue Eyes Intelligence Engineering \& Sciences Publication 
From fig three, the proposed concept is to put into effect this gadget in any educational group. Each visit out of doors the institution can easily discover a holiday spot by way of using this tracking machine. The smartphone will act as a GPS receiver, and he will manual him to his destination imply its function on campus. All of the crucial data can be to be had inside the database of facts. Cell network or wireless canaccess server. Positioning technologies will assist humans to advantage precise region by means of providing precise role data and the purchaser interface to in form. We've installation a hypothetical view on the display of the gadget is implemented at the 4th floor of a building of the institution, wherein the man or woman's function is marked with a crimson dot. The dot will move when it comes to the person's role displayed on the show screen inside the form of a map. [3]

A. Fitness HEAL- tracking in emergency motors with their Authentication with RFID and vicinity monitoring by GPS

Cloud computing is a era this is swiftly based on a virtualization approach in which RFID is some other technology this is used to song objects. By integrating RFID technology with a number of the most important cloud garage, emergency vehicles which includes ambulances, fire engines made by using loose site visitors hold to upward thrust another automobile [4]. RFID tags are located on emergency vehicles scanned through RFID readers positioned at website site visitors alerts. Then the GPS concept (which is also used to tune wherein the automobiles) used within the meantime, we ought to cognizance at the patient's health situations, specifically heart rate, pulse and so on.

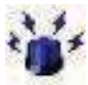

a flashing blue light manifest a volunteer fire fighter retort to an emergency call[4].

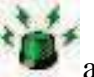

a flashing green light intimate a volunteer ambulance service member react to an emergency call[4].

Yellow flashing light suggests the hazard of the automobile worried in a dangerous operation. (Snow plow) [4].

A. Advanced automobile monitoring and monitoring machine based totally Raspberry P $i$

In the last decade, we observed 'n' quantity of accidents and car robbery sports and greater danger conditions.In this paper really describes an advanced vehicle tracking and tracking structures use an embedded linuxboard.By this system, it is helpful for the safety of youngsters and additionally offer an correct arrival time of the vehicle at a selected vicinity or forestall.It also prevent leakage of LPG fuel.

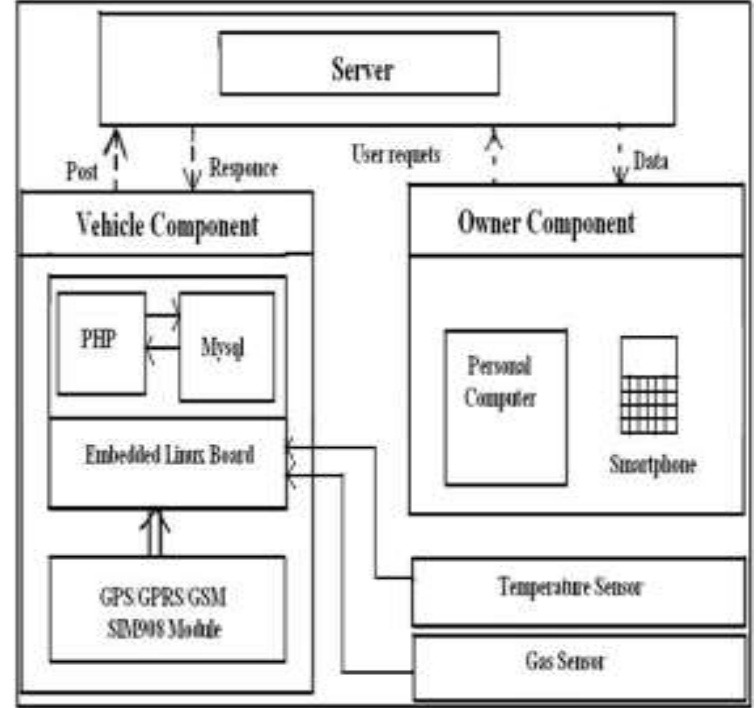

Fig. 5. System Block Diagram

The proposed device would advantage manipulate with the help of Raspberry pi that is positioned within the module SIM908 GSM GPRS GPS automobile.The will get to talk with raspberry pi board with USB interface.The longitude and range of the present day route are received from GPS GPRS GSM module SIM908 advantage in comparison with longitude and variety stored in a selected record layout in the listing raspberry pi.Additionally the longitude and variety of the present day acquired from the GPS might be despatched to the server with the assist of GPRS which allows to song automobiles modern vicinity on a web web web page using the clever telephone.Here to music vehicles, the proposed gadget presents the ability to go surfing the internet page for automobile owners, college students and their dad and mom, as well as the protection of college college students is probably provided with the help DS18B20 temperature sensor and gasoline leakage sensor receives MQ6.Those sensor interface with raspberry pi. If the temperature crosses a positive price or LPG fuel get kebocora $\mathrm{n}$ in the car then a warning message might be despatched to owner.Likewise protection mechanisms furnished with the useful resource of the gadget [5].

\section{CONCLUSION}

From the above evaluate it is smooth that an expansion of techniques available to avoid accidents and car robbery.We have reviewed the one-of-a-kind strategies by way of the usage of special researchers to display screen and monitoring.Typically in all their letters the usage of GPS / GPRS strategies for monitoring variations and vigilant owners and drivers the usage of numerous networks. The ordinary intention of this analysis offers a variety of strategies to at ease the automobile.

\section{REFERENCES}

1. Shiv. H. Sutar, RohanKoul, RajaniSuryavanshi, "smart phone Integration and IOT to the development of smart Public Transportation systems", 2016 international 
conference on net of factors and packages (IOTA) Maharashtra Institute of generation, Pune, India January 22 to January 24, 2016.

2. Md.MarufiRahman, JannatulRobaiat Mou1, Kusum Tara, Md. Ismail Sarkar, "real Time Google Map and Arduinobased vehicle monitoring gadget", 2nd international conference on electrical, laptop and Telecommunication Engineering (ICECTE) eight to 10 December 2016 Rajshahi-6204, Bangladesh.

3. Sunjida Sultana, MahdiaTahsin, Tasmia Reza and Md. Hossam-E-Haider, "An progressive Implementation Indoor Positioning machine using GPS".

4. M.ArunFera, R.Aswini, M.Santhiya, KRGayathiri Deepa, M.Thangaprabha, "HEAL $\square$ health monitoring in emergency motors with their Authentication with RFID and location tracking by means of using GPS" 7th global conference 2015 advanced Computing (ICoAC),

5. A. Prashant Shinde, Prof.Mr.Y.B.Mane, "superior automobile tracking and tracking gadget based completely Raspberry Pi", IEEE sponsored ninth international convention on wise structures and control (ISCO) in 2015. 